\title{
VALOR Y RAZÓN
}

Vamos a discutir en este trabajo la relación entre el valor y la razón desde dos puntos de vista, a saber:

1) La necesidad lógica de la razón en la conducta moral;

2) La relación lógica entre "es" y "debe".

Hemos escogido estos dos problemas porque, por un lado, iluminan con gran claridad la relación entre valor y razón, y, por otro lado, son tratados ampliamente en las discusiones éticas contemporáneas, proporcionándosenos asi la oportunidad de comparar el planteamiento actual del problema -en la axiología material - con el planteamiento del mismo problema en la axiología formal. En aquélla, el planteamiento es filosófico, en el sentido de que procede por la explicitación de implicaciones contenidas en ciertos conceptos claves; en ésta, el planteamiento es científico en el sentido de que constituye la aplicación de un marco de referencia formal al problema o problemas en cuestión. En términos de Hempel, en el primer caso la axiologia solamente tiene un alcance empirico, en tanto que en el segundo caso posee un alcance sistemático-empirico.

Según Hempel, los conceptos con alcance empírico pueden definirse fácilmente en cualquier número, pero la mayor parte de ellos no tendrá utilidad alguna para propósitos sistemáticos; ${ }^{1}$ "ellos no pueden suministrar ninguna comprehensión teórica de los fenómenos en cuestión".2 En la ética, esto significa que la diferencia entre una teoría de alcance meramente empirico y una de alcance sistemático-empírico consiste en que la primera es una explicación ad hoc de un conjunto limitado de fenómenos morales en términos de conceptos analíticos y materiales, sin conexión sistemática con el resto de la filosofia moral, en tanto que la segunda es una representación teórica del conjunto potencialmente ilimitado de la totalidad de tales fenómenos, explicándolos - y realmente determinándolos - sobre la base de una premisa formal. La diferencia es similar a la que existe entre la alquimia y la química. La alquimia era una multitud inconexa de palos a ciegas dirigidos a la naturaleza; del mismo modo, la mayoría de la ética de nuestros días es una multitud inconexa de palos a ciegas dirigidos a la naturaleza moral.

Demostraremos la diferencia entre estas dos clases de procedimientos axiológicos, el material-analítico y el formal-sintético, en relación con los dos problemas fundamentales mencionados. Los dos problemas serán tratados tanto crítica como constructivamente. Las demostraciones en cuestión son

1 Hempel, Fundamentals of Concept Formation in Empirical Science, Chicago, 1952, pág. ${ }^{6}$.

2 Hempel, op. cit., pág. 40. 
experimentos en el alcance sistemático-empírico de la axiología formal, y, de tal suerte, pruebas del método axiológico y de su capacidad de resolver problemas éticos insolutos. Como tales, constituyen verificaciones empírico-morales de este método. Al mismo tiempo, haremos avanzar nuestro conocimiento del asunto discutido: el uso de la razón en la ética. Se advertirá que los dos problemas están interconectados tanto formal como materialmente.

1. La necesidad lógica de la razón en la conducta moral

Veamos primero cómo es considerado este problema en la filosofía actual. Examinaremos una de las discusiones más recientes, el ensayo de Paul W. Taylor, "Four Types of Ethical Relativism". 3 El relativismo ético, desde luego, representa la posición de que los valores son relativos. Taylor resuelve el problema de los criterios en cuyos términos los valores son relativos ofreciendo cuatro tipos de relativismo: 1 ) el relativismo social o cultural (los valores morales son relativos a una sociedad dada), 2) el relativismo psicológico o contextual (los valores morales son relativos a las situaciones en las cuales se producen), 3) el relativismo teórico o lógico (los valores morales pueden ser justificados racionalmente, pero sólo mediante la presuposición del valor de la razonabilidad, lo cual no puede ser justificado), 4) el relativismo metodológico (los valores morales son relativos a los métodos utilizados para juzgarlos). Los dos primeros tipos de relativismo son naturalistas - definen el valor en términos de sociedad y situaciones. Vamos a omitir el carácter naturalista de la bondad de nuestra discusión, como uno de los dos defectos obvios de la ética tradicional (el otro es la indefinibilidad de la bondad no-naturalista). ${ }^{4}$ Pero el tercero y el cuarto son pertinentes a nuestra discusión. Concentraremos nuestra atención en ellos.

\section{(a) El relativismo lógico}

(a) El problema

El problema de éste se reduce a lo siguiente: "Puede darse respuesta a la pregunta ¿por qué ser razonable? sin presumir el valor de ser razonable y, en consecuencia, dar por sentado lo que se discute. El problema tiene gran importancia porque llega al corazón mismo de los ataques que tan violentamente se desencadenan hoy a través del mundo entero contra el intento de vivir una vida racional." 5

3 "Four Types of Ethical Relativism", en The Philosophical Review, LXIII, págs. $500-516(1954)$.

4 Véase Robert S. Hartman, La estructura del valor, Fondo de Cultura Económica, México, 1959, cap. I.

5 Taylor, op. cit., pág. 507. 
Taylor distingue tres sentidos de la pregunta: el pragmático, el moral y el teórico.

En el sentido pragmático, la pregunta significa: “ ¿Es útil o prudente ser razonable en estas circunstancias?" Esta es una pregunta que tiene sentido. "Es posible contestarla en la afirmativa o en la negativa. Es prudente para una persona ser razonable acerca de lo que es bueno para su salud, pero difícilmente podríamos decir que un soldado debe ser razonable con su enemigo en el campo de batalla. Aquí, nuevamente, es perfectamente consecuente usar la razón para justificar el no usar la razón",, 6 , más bien, usar la razón para justificar la utilidad o la prudencia de no usar la razón. Taylor, en una forma tal vez no muy legítima, establece una identificación entre "es útil o prudente" y "es razonable".

En el sentido moral, la pregunta significa: "¿Estoy moralmente obligado a ser razonable en estas circunstancias?", y la pregunta de si hay una obligación moral de usar la razón es, nuevamente, una pregunta que tiene sentido; pues, al justificar nuestra obligación moral de ser razonable, no damos por sentado lo que se discute, es decir, el problema de si uno debe ser razonable a menos que "tener una obligación moral" se identifique con "dar una razón para". Taylor, desgraciadamente, y otra vez en una forma no muy legítima, establece esta identificación y así complica innecesariamente su argumento. Lo que él debió haber dicho es: Yo debo, desde luego, dar buenas razones para la obligación moral de ser razonables, pero éstas son buenas razones para una obligación moral y no buenas razones para dar buenas razones. En consecuencia, no se da por sentado ningún problema en discusión. Podemos muy bien decidir razonablemente que no es nuestra obligación moral ser razonables, por ejemplo, "con un demente escapado del manicomio que está a punto de agredir a mi familia".7 En lugar de eso, Taylor complica el argumento al identificar "estar moralmente obligado" con "dar razón para" y al cambiar la pregunta " $¿$ Estoy moralmente obligado a ser razonable en estas circunstancias?" por "¿He de dar razones para ser razonable?", pregunta que, dice él, puede contestarse dando tales razones. Él complica más el argumento al identificar "ser razonable en estas circunstancias" con "usar procedimientos racionales al tomar decisiones morales y al resolver conflictos morales", 8 y al asignar así el carácter moral, que en su formulación original de la forma "moral" de la pregunta - "Estoy moralmente obligado a ser razonable en estas circunstancias?" - pertenecía a "obligación", y a aquello a que se refiere la obligación, es decir, ser razonable. Aquí tenemos un ejemplo típico del tipo de argumento que abunda en el análisis ético de nuestros días y el cual,

6 Op. cit., pág. 508.

7 Op. cit., pág. 507.

8 Ibid. 
en sus súbitos saltos de significado, evoca poderosamente los argumentos alquímicos de la filosofía natural.

En la interpretación teórica tenemos, finalmente, la clara pregunta: “¿Es razonable ser razonable?’' en principio y überhaupt. Ésta, le parece a Taylor, "es una pregunta muy peculiar. De hecho, es una pregunta que no haría nadie que pensara sobre lo que estaba diciendo, dado que la pregunta, para decirlo con ligereza, se contesta a sí misma. Es admitido que ninguna dosis de argumentación puede hacer que una persona que no quiere ser razonable lo sea. Pues argumentar sería dar razones, y dar razones presupone ya que la persona a la que se le dan está buscando razones. Es decir, presupone que la persona es razonable. Una persona que no quisiera ser razonable en ningún sentido, nunca preguntaría: ‘¿Por qué ser razonable?' Pues al hacer la pregunta ‘¿por qué?’ estaría buscando razones, es decir, estaría siendo razonable al hacer la pregunta. La pregunta reclama el uso de la razón para justificar cualquier uso de la razón, incluyendo el uso de la razón para contestar la pregunta. No se establece ninguna distinción entre un uso de primer orden y un uso de segundo orden de la razón".9

Aquí tenemos otra confusión, porque tal distinción resolvería el problema. No existe ninguna razón por la que en la pregunta teórica concerniente a la razonabilidad del uso de la razón no se haga uso de la distinción de razonabilidad de primer orden y de segundo orden. Definamos la razonabilidad de primer orden como "el uso de la razón" o "el uso de la razón en acción", o, en suma, "juicio racional", y la razonabilidad de segundo orden como "el juicio acerca del juicio racional". Si esto se hace, no es en forma alguna contradictorio, como sostienen los relativistas teóricos según Taylor, o carente de significado, como sostiene el propio Taylor, ${ }^{10}$ preguntar “¿Por qué ser razonable?” y, sin embargo, no ser razonable ni esperar razonabilidad. Pues hay una diferencia de orden lógico entre el hacer una pregunta y el contenido de la pregunta. En particular, hay una diferencía de orden lógico entre el ser razonable al hacer una pregunta y la razón como contenido de la pregunta. Uno no puede ni dar por contestada una pregunta ni contradecirla por la acción de preguntar. La pregunta hecha y el hacer la pregunta están en diferentes niveles lógicos. Cuando hacemos una pregunta, somos razonables o irrazonables del primer orden. Pero el contenido de la pregunta - y de su contestación_ menciona y no usa la razonabilidad o la irrazonabilidad, y así es razonable o irrazonable del segundo orden. En la acción de hacer una pregunta no podemos, por lo tanto, ni dar por sentado ni contradecir el contenido de la respuesta a nuestra pregunta (aunque podemos dar por sentada o contradecir, en algún sentido, la acción de responder). Así, pues, decir que el hacer la pregunta "¿Por qué ser razonable?" podría

9 Op. cit., pág. 509 .

10 op. cit., pág. 5og. 
ser irrazonable, no es una contradicción; pues podemos muy razonablemente (primer orden) preguntar algo irrazonable (segundo orden), y viceversa, muy irrazonablemente (primer orden) preguntar algo razonable (segundo orden).

Por lo tanto, Taylor se equivoca en su caracterización de la pregunta "¿Por qué ser razonable?" Esta pregunta, en su tercera interpretación -la "teórica"-, "es igual a la pregunta ¿En qué consisten las buenas razones para ser razonable? o ¿En qué consisten las buenas razones para buscar buenas razones? El que pregunta está, de tal suerte, buscando buenas razones para buscar buenas razones. La peculiaridad de esta situación se deriva en realidad del hecho de que, en un sentido estricto, la pregunta carece de significado, pues que toda contestación que pudiere aceptarse como una contestación satisfactoria constituiría una tautología al efecto de que es razonable ser razonable. Una contestación negativa a la pregunta ¿Es razonable ser razonable? expresaría una contradicción interna".11 Pues decir: "Es irrazonable ser razonable" contradiría la razonabilidad de decir tal cosa.

Aquí tenemos la confusión entre forma y contenido que hemos discutido en otro lugar; la falacia del método. ${ }^{11 a}$ El decir algo y el contenido de lo que se dice están en diferentes niveles de lenguaje. Por lo tanto, la razonabilidad de decir algo no puede contradecir la irrazonabilidad de lo que se dice, y, a la inversa, la irrazonabilidad de decir algo tampoco puede contradecir la razonabilidad de lo que se dice, por ejemplo, la afirmación a gritos de un demente de que es razonable ser razonable; así como tampoco hay contradicción en la afirmación de Epiménides de Creta de que todos los cretenses son mentirosos: su afirmación de que ellos lo son está en un nivel diferente del de que ellos sean lo que él dice que son."12 Asi, pues, el "relativismo lógico" de Taylor es relativismo sólo cuando cometemos una falacia de tipos. En tal falacia incurren tanto el propio Taylor como los relativistas teóricos o lógicos a los que él se refiere. Según Taylor, la pregunta “¿Es razonable ser razonables?" carece de significado, pues al hacerla somos razonables, y de tal suerte damos la contestación en la pregunta. No podemos, dice el relativista lógico, dar razones para ser razonables, pues el contenido de nuestras razones sólo confirmaría nuestra acción de razonar. Taylor está de acuerdo; pero, dice él, esto no es un argumento contra una justificación tal de ser razonables. Pues

11 Ibid.

11 a Véase La estructura del valor, Introducción y passim.

12 Véase La estructura del valor, pág. 94. Whitehead y Russell, Principia Mathematica, Cambridge, 1935, pág. 62. Pero sí es dar por sentado lo que se discute si el contenido de la pregunta presupone lo que está en discusión. Decir, en una discusión ética, "Lo bueno es el placer", da por sentado lo que se discute, a saber, que lo bueno "es" algo. Decir "Lo bueno es el placer" y luego disfrutar el mal no contradice la proposición "Lo bueno es el placer". Contradice la acción de proponerlo. Las proposiciones pueden ser contradictorias de las proposiciones, por ejemplo, "Lo bueno no es el placer"; y las acciones pueden ser contradictorias de las acciones. Pero las proposiciones no pueden ser "contradictorias" de las acciones. 
tampoco puede hacerse significativamente una demanda a favor -o en contra- de tal justificación. Quien justifica su racionalidad no es ni inconsecuente ni irrazonable. Y nadie puede impugnarle y preguntarle “¿Por qué ser razonable?" sin dar por sentada la pregunta al hacerla. Así, pues, la afirmación del relativista lógico de que nunca podemos justificar el ser razonables - porque la justificación es necesariamente racional y da por sentado lo que se discute - es en sí misma injustificada. Quien da por sentado lo que se discute no es tanto quien justifica su racionalidad como quien le pregunta por qué lo hace.

Este argumento más bien intrincado y algo desorientador - que evoca en esta ocasión no tanto los procedimientos alquímicos como los escolásticoses en realidad bastante impertinente al problema. El problema es si la pregunta “¿Por qué ser razonables?” o “¿Por qué ser racionales?” es una pregunta significativa que puede contestarse significativamente. Como hemos visto, el contenido de la pregunta no debe confundirse con la acción de hacer la pregunta. La contestación al contenido de la pregunta debe estar, pues, en el mismo nivel que el contenido de la propia pregunta, y no en el de la acción de preguntar. La contestación, en otras palabras, debe ser un contenido de una aseveración y no una acción de aseverar; y el uso de la racionalidad o de la irracionalidad en la acción de preguntar o de contestar no tiene nada que ver lógicamente ni con la pregunta ni con la contestación.

\section{(B) La resolución formal}

Veamos entonces cómo contesta la pregunta la axiologia formal. De acuerdo con la axiología formal, cualquier cosa debe cumplir su definición. ${ }^{13}$ La definición de "hombre", suficiente para el propósito actual, es "el hombre es un animal racional". Por lo tanto, el hombre debe ser racional.

Esto es, en realidad, todo lo que hay en este "problema". A cualquiera que nos reproche que esta solución es demasiado fácil y no trata con suficiente profundidad un problema tan profundo, sólo podemos repetir la respuesta de Salvati al reproche similar de Sagredo, y su observación de que la gente tiene en mayor estima lo que adquieren "a través de largas y oscuras discusiones" que lo que adquieren "con tan poco trabajo": "Si aquellos que demuestran con brevedad y claridad la falacia de muchas creencias populares fuesen tratados con desprecio en lugar de gratitud, la injuria seria bien tolerable; pero, por otra parte, es muy desagradable e irritante ver hombres que alegan ser tan duchos como cualquier otro en un cierto campo de estudio, dar por sentadas ciertas conclusiones que más tarde otro hombre demuestra fácil y rápidamente que son falsas... Yo he escuchado, ciertamente, 
de nuestro Académico muchas de esas falacias que se sostienen como verdades pero son fácilmente refutables; algunas de ésas tengo en mente." 14

Es posible, desde luego, complicar el asunto y preguntar: "Pero, ¿̨por qué usar la axiología formal?" La respuesta es: "Porque ella es instrumento del pensamiento más poderoso que la antigua filosofía del valor o el sentido común." La pregunta "Pero, ¿por qué usar un instrumento del pensamiento más poderoso?" se reduce a la pregunta: "Pero, ¿por qué pensar del todo?", que sería aplicable no sólo a la moral sino también a la ciencia natural e impugna toda la empresa humana. La respuesta es otra vez: "Porque somos humanos"; y éste es un hecho difícil de cuestionar.

El asunto podría complicarse, otra vez, en una forma diferente. Podemos preguntar: "En qué consisten las buenas razones para ser razonables?" Ésta es una pregunta muchísimo más complicada que "¿Por qué ser razonable?" Pues, en tanto que la segunda puede contestarse mediante una simple referencia a la definición del que se supone que es razonable, la primera requiere, además, una definición de la razón. Veamos, entonces, cómo se enfrenta la axiología formal a esta pregunta.

Lo que tenemos aquí, además de razonabilidad de primer y de segundo orden, es bondad de primer y de segundo orden. Pues "ser razonables" significa en realidad sostener que la razón es buena. Así, pues, podemos reformular la pregunta como 1 ) " $\mathrm{CCuáles} \mathrm{razones} \mathrm{son} \mathrm{buenas} \mathrm{para} \mathrm{sostener} \mathrm{que} \mathrm{la}$ razón es buena?"; y 2) "¿Por qué debemos sostener que la razón es buena?" o "¿Por qué debemos ser razonables (racionales)?" Para contestar estas preguntas el sistema de la axiología no sólo debe definir "bueno" y "debe", sino también ser capaz de dar razón consecuentemente de la bondad de primer y de segundo orden, es decir, la bondad de algo $x$-digamos, la razón-y la bondad de una razón para sostener que $x$ es bueno.

Discutamos primero la primera pregunta: ¿Qué razones son buenas razones para sostener que $\boldsymbol{x}$ es bueno? Empecemos por descubrir qué queremos decir por buenas razones. Una buena razón, obviamente, es una razón que sigue al buen razonamiento. El buen razonamiento es el razonamiento acorde con ciertas reglas del razonamiento. Por ejemplo, el buen razonamiento matemático es el razonamiento acorde con las reglas de las matemáticas. El razonamiento que nos concierne ahora no es el razonamiento en términos de número, sino el razonamiento en términos de valor. Las reglas del razonamiento en términos de valor, hemos dicho, son las de la axiología. Así, pues, el buen razonamiento acerca de la bondad sería el razonamiento que sigue las reglas de la axiología. Las reglas de la axiología definen la bondad de algo, digamos de $x$. Así, las buenas razones para sostener que una cosa particular $a$ es buena, sería el razonamiento que pruebe si el sostener que $a$ es

14 Galileo, Two New Sciences, Evanston III., 1946, pág. 162. 
buena sigue la definición de bondad dada por el sistema de la axiología. Y aquí tenemos una definición perfectamente buena de una buena razón para sostener que $a$ es buena: hay una buena razón para sostener que $a$ es buena si el sostener que $a$ es buena sigue las reglas del razonamiento de valor o axiología.

Esto corresponde a la buena razón para sostener que dos manzanas y dos manzanas son cuatro manzanas. Si el sostener que dos manzanas y dos manzanas son cuatro manzanas está de acuerdo con el sistema de la aritmética, según el cual dos y dos son cuatro, entonces el sostener que dos manzanas y dos manzanas son cuatro manzanas sigue el buen razonamiento; y se sostiene con buena razón que dos manzanas y dos manzanas son cuatro manzanas.

La definición axiológica de la bondad que es válida tanto para la bondad de $x$ como para la de las razones para sostener que $x$ es bueno, sería: "Cualquier cosa es buena si tiene las propiedades que el sistema de la axiología le atribuye al considerarla buena." Así, $x$ es bueno si el sistema de la axiología le atribuye ciertas propiedades que lo definen como bueno; y una razón para sostener que $x$, teniendo esas propiedades, es bueno, sería buena si el sistema le atribuyese a esa razón también ciertas propiedades que la definen como buena. El problema, entonces, es: cuáles deben ser las propiedades que un sistema axiológico debe asignar a una cosa para definirla como buena, no importa cuál sea la cosa, incluso la razón para sostener que alguna cosa es buena. La respuesta es: "Algo es bueno si cumple su definición" o "Algo es un buen miembro de una clase $C$ si es un miembro de $C$ y tiene todos los atributos de C." 15 Esta definición de bondad es aplicable tanto a la bondad de una cosa $x$ como a la de la razón para sostener que $x$ es bueno. Si a es una razón en una situación, y la definición de razón en una situación es que la razón debe ser situacionalmente eficiente mediante la posesión de las propiedades que Taylor enumera en su sección pragmática, ${ }^{16}$ entonces una buena razón en una situación es una razón que tiene todas estas propiedades, es decir, que es útil, prudente, un medio para el fin situacional, etc. Y $b$ es una buena razón para sostener que $a$ es una buena razón en la situación, si $b$ es un miembro de la clase de las razones para sostener que $a$ es una buena razón en la situación, y tiene todos los atributos de esa clase. La definición de esa clase la hemos dado más arriba, a saber: ser una razón que pruebe si el sostener que $a$ es buena sigue la definición de bondad dada por el sistema de la axiología. Esta última definición, como acabamos de decir, es "Algo es un buen $C$ si es un miembro de $C$ y tiene todos los atributos de $C$ "'. Asi, pues, una buena razón para sostener que $a$ es una buena razón situa-

15 Véase Robert S. Hartman, "A Logical Definition of Value", en Journal of Philosophy, XLVIII, págs. 413-420 (1951). Cf. La estructura del valor, págs. 43 y sigs., 226 y sigs.

16 P. W. Taylor, op. cit., pág. 508. 
cional debe probar si el sostener que $a$ es una buena razón situacional sigue la definición de bondad dada por el sistema. Así, pues, $b$ es una buena razón situacional si 1 ) $b$ enuncia que $a$ es un miembro de la clase de las razones situacionales, 2) $b$ enuncia los atributos que la definición de razón situacional enumera, 3) $b$ enuncia que $a$ tiene todos los atributos que la definición de razón situacional enumera, 4) $b$ enuncia que $a$ es una buena razón situacional según la definición de bondad dada por el sistema de la axiología. El enunciado 4) es una buena razón para sostener que a es una buena razón.

Veamos ahora la segunda pregunta: ¿Por qué debemos ser razonables (racionales)? Ahora necesitamos nuestra definición de "debe". Formulémosla como "Si $x$ es una $C, x$ debe ser un buen $C$ ". ${ }^{17} \mathrm{El}$ valor de la variable $C$ en este caso es "hombre". Asi, pues, "Si $x$ es un hombre, $x$ debe ser un buen hombre". Ahora aplicamos nuestra definición de bondad a hombre. Un hombre particular es bueno si tiene los atributos de la clase de los hombres. Nuevamente usamos " $\mathrm{E} 1$ hombre es un animal racional". Así, la formulación viene a ser: "Si $x$ es un hombre, $x$ debe ser un animal racional", y esto implica "Si $x$ es un hombre, $x$ debe ser racional", quod erat demonstrandum.

(b) El relativismo metodológico

\section{(a) El problema}

Ocupémonos ahora de la cuarta clase de relativismo de Taylor -el relativismo "metodológico"-_, según el cual todos los valores dependen del método de su determinación. Esta determinación puede ser intuitiva o deductiva. Si es intuitiva, el relativista podría preguntar: "¿Cómo adquiere uno esa intuición?"; si es deductiva, podría preguntar: "¿Por qué escoger un sistema y no otro?" Taylor cree que "el reto del relativista metodológico puede ser encarado con éxito sólo de la siguiente manera. La respuesta adecuada a la pregunta ¿Por qué debe utilizarse este método y no aquél, para verificar afirmaciones morales?, consiste sencillamente en que esto, y no aquello, es lo que significamos ordinariamente cuando decimos que una afirmación moral es verdadera (lo que significamos ordinariamente, es decir, cuando no hemos articulado ninguna teoría especial de la ética). En lugar de construir sistemas lógicos o de recurrir a sentimientos intuitivos para justificar creencias morales, supongamos que examinamos los procedimientos y el razonamiento que utilizan realmente, en la vida cotidiana, las personas ordinarias (es decir, quienes no son moralistas o filósofos profesionales) para resolver conflictos morales, para justificar afirmaciones morales y para tomar decisiones morales, y luego explicar (hacer explícitos) los principios o razones implícitos en 
esta utilización". ${ }^{18}$ En otras palabras, la solución de Taylor es la wittgensteiniana. ${ }^{19}$ "Si el relativista metodológico todavíą insiste en preguntar: ‘Por qué escoger este método y no aquél?’ y exige una respuesta a la pregunta “Por qué escoger la explicación como método?" ",20 ¿qué significan "racional", "buenas razones", “razonable”?; entonces, dice Taylor, respondemos: "Significan lo que significamos ordinariamente al utilizar los términos 'racional', 'buenas razones' y 'razonable’. ¿Por qué habrian de significar otra cosa? La explicación es sencillamente el proceso mediante el cual este significado ordinario se pone de manifiesto y se hace preciso. Si entonces se pregunta 'Pero, zpor qué buscar una manera racional de resolver una disputa, o buenas razones para justificar creencias morales, o una manera razonable de tomar una decisión moral?', la respuesta es que lo hacemos porque ello constituye un problema en la vida práctica. Lo cierto es que las personas tratan de descubrir la manera de ser razonables en cuestiones de ética. Y la explicación les aclara qué significa ordinariamente, es decir, qué significa para ellas ser razonables en tales asuntos." 21 Taylor aquí aduce al "uso común". Pero Bertrand Russell ha puesto al descubierto, en muchas ocasiones, este fetiche del uso común, que en el presente contexto significa considerar como moral, razonable, racional, lo que personas aun inmorales, irrazonables e irracionales significan cuando dicen cosas inmorales, irrazonables e irracionales llamándolas "morales", "razonables" y "racionales".

\section{(B) La resolución formal}

Veamos ahora cómo encara este problema la axiología formal. "¿̨Por qué escoger un sistema de valor y no otro?" Esto es, obviamente, un problema de valoración. De aquí que los sistemas de valor en cuestión deban contener reglas para resolverlo, es decir, deban contener reglas para escoger cualquier cosa, incluidos los sistemas de valores. Si uno de los sistemas de valor en cuestión no tiene tales reglas, está ya descalificado; pues, obviamente, un sistema de valor que no contiene reglas para escoger -incluido el escoger sistema de valor - no es un buen sistema de valor. Si otros sistemas de valor en cuestión contienen tales reglas, son mejores sistemas de valor y deben ser escogidos. Si ninguno de los sistemas de valor contiene tales reglas, ninguno es bueno y ninguno debe ser escogido. Si todos contienen tales reglas, estas reglas deben ser comparadas. De ahi que el problema se reduzca a las reglas de comparación contenidas en los sistemas de valor en cuestión, y la aplicación de esas reglas de comparación a las reglas de comparación de los sistemas.

18 Taylor, op. cit., pág. 514 .

19 Véase La estructura del valor, págs, 160 y sigs.

20 Taylor, op. cit., pág. $5^{16}$.

21 lbid. 
Según la axiologia formal, estas reglas se derivan de la definición de "mejor". Una cosa $A$ es mejor que una cosa $B$ si $A$ contiene más propiedades de clase que $B .{ }^{22}$ También, de acuerdo con la axiología formal, debemos escoger lo que es mejor y no lo que es peor. ${ }^{23}$

Aplicado a un sistema de valor, esto significa que un sistema de valor es el mejor mientras más satisfaga la definición de un sistema de valor. Debemos, por lo tanto, definir el criterio o los criterios que hacen, o cuya ausencia deshace, una teoría del valor.

Una teoría del valor, obviamente, debe ser una teoria del valor, es decir, debe ser una teoría que explique el mundo del valor. Cualquier teoría que sea llamada una teoría del valor pero que no explique el mundo del valor, no es una teoría del valor. El mundo del valor es la totalidad de los fenómenos de valor. Por lo tanto, el criterio singular que hace o deshace una teoria del valor es el de la aplicabilidad universal. Una teoría que sea llamada una teoría del valor y no sea universalmente aplicable no es, según este criterio, una teoría del valor.

De tal suerte, así como la prueba de un pastel radica en ser comido, la prueba de una teoría del valor radica en ser aplicada. Hay un modo elegante y un modo menos elegante de llevar a cabo esta prueba. El modo elegante consiste en investigar la analiticidad o sinteticidad de la teoría del valor. La "mejor" teoría del valor, desde el punto de vista de la aplicabilidad, será una teoría formal o sintética, y, ciertamente, ninguna teoría del valor que no sea formal o sintética será, de acuerdo con esta prueba, una teoría del valor. Esto se desprende de la naturaleza misma de las teorias sintéticas en contraste con las teorías analíticas. ${ }^{24}$

Pero este modo elegante es accesible únicamente a las mentes adiestradas en la filosofía y la ciencia. El modo menos elegante consiste en enumerar todos los fenómenos de valor a los cuales una teoría es aplicable, y eliminar de la lista de las teorías del valor a cualquiera de ellas que no explique todos los fenómenos de valor enumerados. Si hay (siendo todas las demás cosas iguales) sólo un fenómeno de valor que la teoría del valor $A$ no explica, en tanto que la teoría $B$ sí lo explica, entonces la teoría del valor $A$ no es una teoría del valor en este caso.

Además del criterio extensivo de la aplicabilidad, podemos admitir algunos criterios comprensivos de una teoría del valor. Así, entre dos teorías igualmente aplicables, será mejor aquella que sea más consecuente; y entre dos teorías igualmente consecuentes, será preferible la que sea más elegante, es decir, la que logre su resultado con los medios más económicos y sencillos (principio de la parsimonia). En la ciencia natural fue preciso recu-

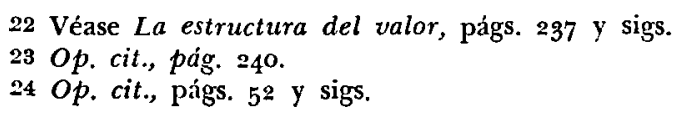


rrir a este último criterio para determinar los méritos relativos de la teoría copernicana y la ptolemaica, puesto que en su tiempo ambas eran igualmente aplicables y consecuentes. La mayor elegancia de la teoría copernicana se derivaba de su mayor abstracción. Pero, puesto que el criterio de la abstracción y la elegancia es, por una parte, accesible únicamente a las mentes especialmente adiestradas $y$, por otra parte, conduce a primera vista a resultados absurdos, la aceptación de esta teoría fue lenta y tortuosa. En la teoría del valor, dado que no tenemos aún muchas teorías igualmente consistentes y aplicables, no tenemos que recurrir todavía a este último criterio. Prácticamente es probable que la pregunta del "relativista metodológico" de Taylor nunca se produzca; pues, como observaba Einstein en el caso de la ciencia natural, ${ }^{25}$ sólo habrá una teoría que explique el campo entero del valor. Tal teoría, por supuesto, debe ser capaz de explicar un fenómeno de valor tan pertinente y tan común como el valor de una teoría del valor. La aplicabilidad de una teoría del valor a la filosofía en general, y a la teoría del valor en particular, es un criterio para la validez de una teoría del valor. La aplicación de la teoría del valor a la teoría del valor es, por lo tanto, una piedra de toque para ambas teorías.

El "relativista metodológico" de Taylor, al hablar de la valoración de las teorías del valor, deja de tomar en cuenta el carácter mismo de estas teorías como teorías del valor. Ello se debe a que utiliza nociones tales como “elección", "valor", “teoría", etc. en su vago significado analítico, no pudiendo asi comprender cabalmente aquello de que habla: que las teorías del valor deben contener la solución del problema que él está planteando. El argumento entero, por lo tanto, es impertinente al problema. La pregunta "¿Por qué debe utilizarse este método y no aquél para verificar afirmaciones morales?" o "¿Por qué escoger un sistema de valor y no otro?", depende de la definición de "debe", "escoger", "mejor", etc. en los respectivos sistemas de valor. Así, pues, la pregunta del "relativista metodológico" carece de significado. $\mathrm{O}$ bien los sistemas en cuestión son teorías del valor, en cuyo caso contendrán reglas para dar respuesta a la pregunta (y la pregunta carecerá de significado porque su respuesta está dada con el sistema), o bien no son teorias del valor, en cuyo caso la pregunta no tiene sentido, pues no se refiere a ellas. De tal suerte la axiologia formal demuestra la carencia de significado de la pregunta que Taylor considera llena de significado - la del relativista metodológico-, y como llena de significado la pregunta que Taylor considera carente de significado _la del relativista lógico.

Nuestra respuesta a la pregunta relativa a la necesidad lógica de la razón en la conducta humana es que esta necesidad se deriva lógicamente de las reglas de la axiología formal cuando son aplicadas a los seres humanos. Examinemos ahora desde otro ángulo el papel que desempeña la razón en la moralidad.

25 Op. cit., pág. 140. 


\section{La relación lógica entre "es" $y$ "debe"}

Ahora veremos cómo la definición de "es" y "debe" encaja en la discusión contemporánea de la relación entre valor y razón. Haremos una comparación entre un tratamiento analítico del problema, a la manera de la filosofía moral tradicional, y el tratamiento sintético de la axiología formal. Escogeremos un texto reciente y particularmente ingenioso, de A. Campbell Garnett."26 Mientras que la axiología formal deriva el "debe" del "es", mediante la equivalencia de "debe" con los diversos significados de la relación formal "es menor que", Garnett encuentra el "debe" en una implicación material del concepto analítico "razonable".

El problema de Garnett consiste en cómo dar razón de la normatividad de los juicios de valor, no por implicación, como cuando el valor es definido en términos normativos - "ley", "debe", "deber"-, sino cuando el valor es definido en términos no-normativos, tales como "bueno".

El problema es: cómo puede "bueno" ser empíricamente definido de tal manera que la definición, como parte de una primera premisa en un silogismo, pueda proporcionar una conclusión normativa. La solución de Garnett es ingeniosa. " $x$ es bueno" es equivalente a " $x$ es un objeto razonable de una actitud favorable". Ésta es una definición no-normativa y empírica, en términos de la inteligencia o de la razón. Ahora bien, dice Garnett, aunque “el término 'bueno' no implica, de por sí, un concepto normativo. . a a través de la palabra 'razonable' entra en la definición una implicación material de normatividad; pues generalmente se presume que 'uno debe ser razonable' ". Por lo tanto, si $x$ es un objeto razonable de una actitud favorable, entonces $x$ debe ser hecho el objeto de una actitud favorable. Todo el mundo tiene la obligación de usar su inteligencia. "El principio de que uno debe ser razonable, firmemente establecido como presunción básica, lleva así a la mente de ' $x$ es bueno' a ' $x$ debe ser el objeto de una actitud favorable" ",27 mediante una "transición natural" dada en el "silogismo implícito":

Lo que es un objeto razonable de una actitud favorable debe ser hecho el objeto de una actitud favorable.

$x$ es bueno, es decir, es un objeto razonable de una actitud favorable.

Por lo tanto,

$x$ debe ser hecho el objeto de una actitud favorable.

Así, pues, aunque la obligación de ser razonable no está establecida por

26 The Moral Nature of Man, New York, 1952, págs. 112 y sigs. “A Non-normative Definition of Good", en The Language of Value, Ray Lepley, ed., New York, 1957, págs. 122 sigs.; "Is Good a Normative Concept?", en Philosophical Quarterly, VII, págs. 260-263 (1957).

27 A. C. Garnett, Philosophical Quarterly, op. cit., pág. 26g. Cf. Kant: "Lo que llamamos bueno debe ser, a juicio de todo hombre razonable, un objeto de la facultad del deseo." (Critica de la razón práctica, parte I, libro I, cap. II.) 
la definición de "bueno", y ni ésta ni ninguna otra obligación es afirmada por el uso de este término, sí surge una afirmación de obligación por la razón de "una proposición sintética independiente que presume la gente que usa el término". La afirmación de razonabilidad no es normativa, pero generalmente se presume que lo es. La definición de Garnett alega "clarificar el uso ordinario y señalar por qué generalmente se entiende que los enunciados acerca de lo que es 'bueno' implican proposiciones que contienen un 'debe'". La definición de "bueno" de Garnett es una definición empírica concebida para mostrar ese elemento — "razonable" - que implica materialmente a "debe".

Bien se puede convenir con Garnett en que la definición de "bueno" contiene un elemento que implica un "debe". Pero este enunciado -que la definición de "bueno" contiene un elemento que implica un "debe"- es un enunciado formal, que trata de una definición, y no hay razón por la que deba ser expresado en términos empíricos. En realidad, tal expresión priva a la definición de su poder formal. Pues ninguno de sus términos empíricos - ni "objeto de una actitud favorable" ni "razonable"- es necesario a fin de producir la implicación material de la normatividad. El primer término - "objeto de una actitud favorable" - es innecesario, pues la implicación se adhiere a "razonable". El silogismo sería igualmente válido si escribiéramos:

Lo que es un $A$ razonable debe ser un $A$

$x$ es un $A$ razonable

Por lo tanto

$x$ debe ser un $A$

Si queremos definir "un $A$ razonable" de tal modo que " $x$ es un $A$ razonable" sea equivalente a " $x$ es bueno", podemos usar ahora las diversas definiciones empíricas de "bueno" propuestas en la literatura y sustituir " $A$ " por cualquiera de ellas: "objeto de placer", "objeto de deseo", "objeto de satisfacción", "objeto de aprobación", "objeto de interés", "objeto de intención", etc., cualquiera de éstas serviría para el caso, y existen tantas "buenas" razones para que Garnett use su definición para "bueno" - razones que son "objetos razonables de una actitud favorable"- como existen "buenas" razones para que otros éticos usen sus definiciones de "bueno" -razones que son "objetos razonables de placer", "objetos razonables de satisfacción", etc., para ellos.

Pero también la otra parte de la definición de Garnett - la palabra "razonable" - comparte la arbitrariedad de todas las definiciones empíricas de "bueno". Garnett enuncia que "se presume que uno debe ser razonable". ¿Qué tal si otro ético dijera que se presume que uno debe ser complacido? ¿O satisfecho? ¿O intencionado? En otras palabras, ¿no serían igualmente válidas, para la presunción del "debe", cualesquiera otros de los elementos empíricos de la definición de "bueno"? Uno podría muy bien decir que el ser razonable no cuenta nada si uno no tiene un objetivo; puesto que, como 
dice el propio Garnett al refutar a G. E. Moore, “debe” se refiere a las acciones humanas responsables. Así, pues, es más bien intencionado que razonable lo que se presume que debe ser todo el mundo. También, podríamos decir, preferimos la satisfacción a una actitud favorable como la característica de lo bueno. Por lo tanto, definimos " $x$ es bueno" como " $x$ es un objeto intencional de satisfacción", y decimos "todo lo que es un objeto intencional de satifacción debe ser un objeto de satisfacción". Entonces, si $x$ es bueno, es decir, un objeto intencional de satisfacción, la conclusión es que $x$ debería ser hecho un objeto de satisfacción. $O$, nuevamente, podemos usar las dos características de "bueno" en el orden inverso y definir "es bueno" como " $x$ es un objeto satisfactorio de intención", presumiendo que todo el mundo debe ser satisfecho. En otras palabras, la arbitrariedad del nivel empírico se adhiere también a esa característica de "bueno" a la que, según Garnett, se adhiere la presunción de normatividad: cualquier característica semejante puede implicar normatividad. La definición, entonces, viene a ser " $x$ es bueno" es equivalente a " $x$ es $\varphi$ y $x$ es $A$ ", en donde está implicado que si $x$ es $\varphi, x$ debe $\operatorname{ser} A$.

Pero esto es muy similar a la definición que se da en la axiología formal. ${ }^{28}$ De acuerdo con esta definición, $x$ es un buen $A$ si $x$ tiene todas las propiedades $\gamma, \delta, \varepsilon$, o, en general, $\varphi$ (siendo $\varphi$ el variable del cual $\gamma, \delta, \varepsilon$ son los valores). Entonces, si $x$ tiene todas las propiedades $\varphi, x$, de acuerdo con nuestra definición, debe ser $A$, en donde "debe" es el debe formalmente analítico; ${ }^{29}$ si Juan es varonil en todo respecto, debe ser un hombre. Y ciertamente, si él es de tal suerte varonil, será un buen hombre, en este sentido de "hombre": un buen especimen de la hombría. Si, en cambio, Juan no tuviera todas las propiedades de la hombría, es decir, si no fuera varonil en todo respecto, entonces no debería ser un hombre en este mismo sentido. Aquí, "debe" es nuevamente analítico porque Juan en realidad no es un hombre en este sentido. Por otra parte, aunque Juan no es varonil, debería ser varonil en todo respecto. Aquí, "debe" es sintético, pues Juan no es varonil en todo respecto y, por lo tanto, debería ser lo que no es. Como se ve, "debe" se deriva aquí, analítica o sintéticamente, de una definición formal no-normativa de "bueno". La definición de Garnett es'de la misma naturaleza general, pero no está enunciada en términos generales sino particulares, no en términos sintéticos sino analíticos.

Asi, pues, tenemos en total cuatro casos: dos para " $x$ es bueno" (I y II) $y$ dos para " $x$ no es bueno" (III y IV) de la siguiente manera:

I. " $x$ es $\varphi$ y $x$ es $A$ ", en donde " $x$ es $\varphi$ " implica materialmente que "x debe ser $A "$. (Versión formal de la definición de Garnett.)

28 Véase La estructura del valor, págs. 227 y sigs.

29 Véase op. cit., págs. 240 y sigs., 246 y sigs. 
II. " $x$ es $\varphi$ y $x$ es $A$ ", en donde " $x$ es $\varphi$ " implica formalmente que " $x$ debe ser $A$ ".

III. " $x$ no es $\varphi$ y $x$ no es $A$ ", en donde " $x$ no es $\varphi$ " implica formalmente que " $x$ no debe ser $A$ ".

IV. " $x$ no es $\varphi$ y $x$ es $A$ ", en donde " $x$ no es $\varphi$ " implica materialmente que " $x$ debe ser $A$ ".

Como se ve, el tratamiento tradicional del problema por parte de Garnett, y su uso de conceptos analíticos tales como "razonables", "debe", etc., ocultan la naturaleza lógica de su argumento, la cual es clarificada por el procedimiento sintético de la axiología formal. Aqui, "debe" se deriva de "es mejor que", lo cual a su vez se deriva de la definición de "bueno" como cumplimiento comprehensional. El puente entre "es" y "debe" lo constituyen, pues, los términos "bueno" y "mejor": "Es mejor para $x$ ser bueno y no malo" equivale a " $x$ debe ser bueno y no malo". Ninguna determinación empírica es necesaria; por el contrario, la presunción garnettiana de que uno debe ser razonable, puede deducirse de la definición axiológica de "bueno" y "debe" como un teorema. Garnett, al igual que tantos empíricos, coloca la carreta empírica delante del buey teórico. ${ }^{30}$

Aunque el argumento de Garnett puede ser interpretado - y corregido-

30 La deducción axiológica también salva al argumento de la falacia naturalista de G. E. Moore, que causa cierta dificultad a Garnett (The Moral Nature of Man, pág. 120) . El la resuelve, como muchos empiristas, afirmando que no hay tal falacia. El argumento de Moore, dice él, descansa en la identificación de "bueno" con "debe existir", y "debe existir" no significa nada o bien significa algo naturalista ("debe ser favorecido", etc.) -significados éstos que no son, desde luego, ninguno de los dos, lo que el propio Moore quiere decir. En la axiología formal, "debe existir" sí significa algo, y ese algo es nonaturalista. "Debe" puede tomarse, o bien (I) analíticamente, o (II) sintéticamente. Tomado (I) analíticamente, significa sencillamente implicación lógica: para que una cosa sea algo - por ejemplo, buena_ debe scr una cosa. Tomado (II) sintéticamente - éste es el significado de Moore-, significa que una cosa debe ser más bien que no ser si es que ha de ser buena. Esto significa, axiológicamente, que la existencia de una cosa es mejor que su no-existencia, y esto, de acuerdo con la definición de "mejor" (Estructura del valor, págs. 237 y sigs.) significa que una cosa existente tiene más propiedades que una cosa noexistente, lo cual es indudablemente cierto. Puesto que, de acuerdo con (I) una cosa no puede ser buena a menos que sea una cosa, y de acuerdo con (II) debe ser una cosa más bien que no ser una cosa, el que una cosa sea buena significa que debe existir. La corazonada de Moore tiene, pues, una base axiológica; implica mucho más de lo que él ve, y ciertamente mucho más de lo que sus criticos naturalistas ven. En particular, Moore no hace clara la diferencia entre "es bueno" y "es bueno que". " $x$ es bueno, significa que ' $x$ debe existir'" $y$ es diferente de "Es bueno que $x$ exista". La segunda es equivalente a "Es bueno para $x$ existir" o "La existencia de $x$ es buena para $x$ ". De acuerdo con la definición de "es bueno que" (ibid.) esto significa que la comprehensión de "la existencia de $x$ " coincide parcialmente con la comprehensión de " $x$ ", y esto, una vez más, es induda. blemente cierto. Además, arroja luz sobre discusiones famosas en la historia de la filosofía. (Para un tratamiento no-empírico de "existencia", "debe" "bueno", véase Edwin T. Mitchell, A System of Ethics, New York, 1951; págs. 103 y sigs. Para la distinción de Moore entre “Esta cosa existente es buena" y "Esto sería bueno si existiera", véase G. E. Moore, Principia Ethica, México, 1959, pág. 113.) 
por la axiología formal, este argumento no puede, a su vez, interpretar, ni corregir, ni siquiera conducir a tal axiología. La razón de ello es que los conceptos analíticos y materiales no contienen la precisión de significado que poseen los sintéticos y formales; y no hay un puente que lleve de los unos a los otros. Uno tiene que saltar, dejando atrás todo el pensamiento analítico, abstractivo y categorial, y entrar incondicionalmente en el pensamiento sintético axiomático. El argumento analítico, mientras más lejos procede, se hace más complicado y más falso respecto de la realidad material. Al final, el pensamiento se pierde en un laberinto sin salida; y si se aplica la lógica formal a este substrato laberíntico, la confusión es aumentada por el propio poder del simbolismo. El absurdo simple viene a ser entonces absurdo de gran potencia, como hace claro una ojeada al simbolismo astrológico y alquímico. Pues lo que es cierto hoy de los argumentos analíticos en la filosofía moral, era cierto para la misma clase de argumento en la filosofía natural. Sólo que en el caso de esta última, educados como estamos por trescientos años de ciencia natural, una ojeada a un texto de alquimia o de astrología nos revela de inmediato su absurdo. En el caso de la primera, como todavía no tenemos una alternativa científica somos incapaces de reconocer su falsedad. En realidad, somos proclives a confundir lo intrincado con lo profundo; y sólo podemos aguzar nuestro juicio si saltamos y reconocemos la profundidad como un fracaso de comprensión. ${ }^{31}$ Un argumento analítico es más plausible mientras menos se le desarrolla, y se hace menos plausible mientras más se le desarrolla. ${ }^{32}$ Un argumento sintético, por el contrario, se hace plausible mientras más se le desarrolla y es menos plausible mientras menos se le desarrolla. Pues sus postulados, simplemente enunciados, no tienen conexión aparente con la realidad; solamente su elaboración muestra el alcance de su aplicabilidad práctica. ${ }^{33}$

El argumento de Garnett está relativamente poco desarrollado y por eso es bastante plausible. Procedamos, entonces, a desarrollarlo y veamos adónde conduce. Empecemos con su definición. " $x$ es bueno" se define como " $x$ es un objeto razonable de una actitud favorable". Ésta es una típica definición analítica, es decir, una definición que contiene conceptos analíticos. Para entenderla, tenemos que definir los conceptos que hay en ella. Garnett hace esto de la siguiente manera: "Por una actitud 'favorable', queremos decir aquí una actitud inclinada a guardar, conservar o promover la cosa, o la clase de

31 Véase E. Husserl, Philosophie als strenge Wissenschaft, en Logos I, págs. 289-341 (1911). La estructura del valor, pág. 164 .

32 Buenos ejemplos son A. L. Hilliard, The Forms of Value, New York, 1950, y, especialmente, E. W. Hall, What Is Value?, New York, 1952.

33 Por esta razón, la filosofía moral actual se presta para artículos más que para libros; y el llamado de Frankena a los filósofos morales para que escriban más bien libros que artículos (Philosophical Review, XL, pág. 55 (1951), tiene un significado más profundo que el que él mismo pensó. 
cosa, en cuestión. Al decir que una actitud es razonable, queremos decir que la actitud es como la que surgiria de la comprensión iluminada del objeto y de nuestro propio ser y de la relación del objeto con nuestro propio ser." 34 Esto conduce a Garnett a una nueva formulación de su definición: “ ' $x$ es bueno' quiere decir que ' $x$ es un objeto hacia el cual la comprensión iluminada tiende a desarrollar una actitud favorable" ".35 En realidad, Garnett no ha utilizado sus definiciones completas de "favorable" y "razonable" en esta reformulación. Una formulación completa sería “' ' $x$ es bueno' quiere decir que ' $x$ es un objeto hacia el cual la comprensión iluminada del objeto, de nuestro propio ser y de la relación del objeto con nuestro propio ser tiende a desarrollar una actitud inclinada a guardar, preservar o promover la cosa, o la clase de cosa, en cuestión'". El próximo paso, entonces, tendría que ser un análisis de "iluminado" y "comprensión"; luego, un análisis del propio ser y de la comprensión iluminada de éste; después, del objeto y de la comprensión iluminada de éste; finalmente, de la relación entre el objeto y el propio ser y de una comprensión iluminada de esta relación. Y esto implica no sólo, como sostiene Garnett, un estudio de psicología - al cual quiere él reducir la ética, en vez de dejarla que continúe "errando en la estratósfera de las propiedades no-naturales y en la selva de los significados no-cognitivos" 30 sino también un estudio de epistemología (la relación entre el sujeto y el objeto), de ecología (la relación entre el sujeto y el medio ambiente), de historia y educación (la naturaleza de "iluminado"), y de toda clase de ciencias naturales, como la química, la física, la educación, la refrigeración, mantenimiento, etc., dependiendo de cuál sea la cosa a guardarse, conservarse o promoverse. Todo esto debe ser desarrollado, pero no hay ninguna regla dada sobre la manera en que esta tremenda masa de material debe organizarse y diferenciarse para que se haga ética. Más bien, todo se deja tal como está; el pensamiento no tiene dirección para guiarse en este caso y para aplicar a la realidad lo que esta definición significa; de tal suerte que el guardar un bote de mermelada en el refrigerador estaría plenamente de acuerdo con la definición. Ello sería, por lo tanto, una acción moral basada en un objeto moral. Así, pues, la definición es ciertamente demasiado amplia.

Pero en realidad es falsa. Pues hay muchos casos en que un objeto cae dentro de ella - como un objeto razonable de una actitud favorable - y, sin embargo, no es ni bueno ni malo; o en que se toma una actitud irrazonablemente favorable hacia un objeto que es bueno o razonablemente favorable hacia uno que es malo. Hay casos, en otras palabras, en que la gente dice " $x$ es bueno", pero $a$ ) es irrazonable adoptar una actitud favorable hacia $x$,

34 A. C. Garnett, The Moral Nature of Man, pág. 120.

35 lbid.

36 Philosophical Quaìterly, op. cit, págs. 262 y sig. 
o b) es razonable adoptar una actitud desfavorable hacia $x$, o $c$ ) la razón no está en discusión en absoluto.

a) Supongamos que un adúltero habla de su amante y dice: "iQué buena es!", pero él ama a su esposa y sabe que es irrazonable adoptar una actitud favorable hacia su amante. $O$ tomemos por ejemplo a Dean Acheson, exSecretario de Estado de los Estados Unidos, que dice: "Alger Hiss es bueno. Yo no le negaré mi apoyo", pero sabe, sin embargo, que en vista del veredicto contra Hiss como traidor, es irrazonable adoptar semejante actitud favorable hacia éste. O tomemos a Romeo y Julieta, que se aman pero saben que su amor es irrazonable. Ciertamente, cualquier caso en que una persona considere buena cualquier cosa "contra su mejor juicio" - y éstos son los casos profundamente morales- pertenece aquí. Desde luego, Garnett podría decir que en tales casos la gente no quiere decir realmente que $x$ es bueno. Pero ciertamente parece que sí lo quieren decir.

b) "Es mejor ser listo que bueno." Aquí se presume que es razonable adoptar una actitud desfavorable hacia lo que es bueno. "Habrá más gozo en el cielo de un pecador que se arrepiente, que de noventa y nueve justos, que no necesitan arrepentimiento"; aquí tenemos el mismo caso. Confucio dijo: "Ved estos hombres buenos; yo los odio", porque ellos eran tan buenos que no veían el mal y no hacían nada para combatirlo.37 "Ella es en verdad una buena muchacha; no tengo nada que hacer con ella", dice Don Juan y todos los que son como él. "Él no sirve para nada, pero yo lo amo." "Él es malo, pero adorable." Si $x$ no es bueno, ello debe significar que cs razonable adoptar una actitud desfavorable hacia $x$. Pero éste, a menudo, no es el caso. "Lo quiero porque es tan malo." "Éste es su mejor libro, tan malo como todos los demás." "Vamos a ser malos juntos." En otras palabras, hay muchos casos en los que lo que es bueno se considera razonablemente como objeto de una actitud favorable. "Bueno" se utiliza a menudo para expresar una actitud desfavorable, como en la expresión "ibuena porquería!" o en el veredicto de Churchill sobre cierto pintor: "Un excelente ejemplo de arte moderno" (que él detesta), o este juicio que se ha hecho sobre Matisse: "Un buen decorador de interiores", y éste sobre Eisenhower: "Un buen jugador de golf." Existen, en otras palabras, y como lo hace claro la axiología formal, varios niveles de bondad, algunos de los cuales pueden ser malos en términos de algunos otros. Siempre que un concepto $B$ representa un conjunto de propiedades que no cumplen un concepto $A$, entonces, en términos de $A, B$ es malo. Pues, decir que $x$ es un buen $B$ es decir que $x$ es un mal $A .{ }^{38}$ De esta manera, "bueno" puede usarse sistemáticamente como un término general de condenación.

37 Los hsiangyuan, bonachones, que obran "según una conciencia que no es la suya propia" y son los "ladrones de la virtud" (Analectas, cap. xvII).

38 Véase Robert S. Hartman, Axiologia formal: la ciencia de la valoración, Seminario de Problemas Científicos y Filosóficos, Cuaderno 10, México, octubre 1956, pág. 13. 
Además, los diversos niveles de valor que distinguimos -el valor formal, el valor fenomenal y el valor axiológico- pueden hallarse en contradicción entre si. Así, el bien de una persona puede ser el mal de otra. "iQué sano es el invierno!", dice Thoreau, "iqué bueno, por encima de toda bondad moral meramente sentimental, cálida, efímera y tierna...! Yo considero, en el fondo de mi alma, que la mayor parte de lo que mis vecinos consideran bueno, es malo; y si de algo me arrepiento, es muy probable que sea de mi buen comportamiento". ${ }^{39}$ Apenas es posible una actitud desfavorable a la bondad moral más franca que ésta, que de ninguna manera se contradice a sí misma, como sostiene Garnett.40 Ni siquiera seria contradictorio si, por "bondad moral", el que habla significara algo que él mismo - y no otrosllaman así. Obviamente, nuestro rechazo de lo que nosotros mismos llamamos "bueno" es un profundo fenómeno axiológico. Excluirlo de la axiología significaria extirpar de la teoria del valor uno de los más significativos fenómenos de valor. En este sentido, podemos considerar como la refutación final de la teoría del valor emotiva, la siguiente frase de Oscar Levant: "Yo no tomo licor. No me gusta. Me hace sentir bien." 41

La separación de las palabras y los significados, por una parte, y las actitudes y las acciones por otra, constituye uno de los rasgos más característicos de la vida y de la historia humanas. Al combinar las dos cosas, en principio y en la base misma de su argumento, la teoría ética no sólo incurre en la falacia del método, sino que al hacerlo se priva de la comprensión penetrante y pertinente de una buena parte de la realidad moral. ${ }^{42}$

c) Cuando decimos que el caramelo tiene buen sabor, no presumimos ninguna razonabilidad de parte de nadie hacia este caramelo. Meramente declaramos que tiene buen sabor o que es bueno porque tenemos esa sensación en ese momento. Cuando una madre ve a su hijo ahogándose y se lanza al agua aunque no sabe nadar y se ahoga también, no hay en ella ninguna razón, sino meramente instinto, aun si antes de saltar exclamara: "Tengo una actitud favorable hacia mi niño!" El caso es el mismo de la famosa escena, a menudo escrita, en que una pareja resiente cualquier intromisión de fuera, y la famosa frase: "iA nadie le tiene que importar que mi marido me pegue!" Esta puede ser una actitud razonablemente favorable hacia algo malo, o una actitud irrazonablemente favorable hacia algo bueno, o más probablemente ninguna de las dos, sino sencillamente la naturaleza hablando su lenguaje misterioso pero bien fundado. ${ }^{43}$

39 The Living Thoughts of Thoreau, Theodore Dreiser, ed., Filadelfia, 1939, pág. 71.

40 The Moral Nature of Man, pág. 120; Philosophical Quarterly, op. cit., pág. 262.

41 Time, 5 de mayo de 1958 .

42 Cf. la caricatura en The New Yorker, donde aparecen dos niños cuáqueros enfrentados en actitud hostil y gruñendo: "Mi papá es más bondadoso que el tuyo." Cf. La estructura del valor, pág. 191.

43 La axiología formal interpreta todos estos casos como transposiciones de valor, las 
Hay una infinidad de casos en los que la definición de Garnett demuestra ser incorrecta. Ésta es demasiado lata $y$, por lo tanto, inadecuada para cubrir las sutilezas del pensamiento y la acción morales. Con todo, es una de las refinadas definiciones analíticas de "bueno" que existen. Esto demuestra que los conceptos materiales son insuficientes para dar razón de la moralidad. Es necesario desarrollar un concepto más fino, más flexible y más preciso; el concepto formal.

La axiología formal mediante un procedimiento formal nos contesta preguntas que el procedimiento material o filosófico deja en la vaguedad. El procedimiento formal no es más sencillo absolutamente, pero sí es más sencillo relativamente -comparado con su poder explicativo o su precisión- que el procedimiento filosófico. El hecho de que sea más preciso y de que no sea más sencillo tiene la misma razón: que la comprehensión sintética crece —en vez de disminuir - con la generalidad del asunto en cuestión.

Este asunto, en el presente caso, era el valor de la razón para valorar.

Robert S. HARTMAN

cuales son exactamente definidas. La mujer que disfruta los golpes que le da su marido, por ejemplo, valoriza intrinsecamente la desvalorización intrínseca de un valor intrínseco, expresado simbólicamente como " $\left(I_{T}\right){ }^{I}$ ". Para mayores detalles, véase Robert S. Hartman, "The Science of Value", en New Knowledge in Human Values, Abraham Maslow, ed., New York, 1958, y "Value Theory as a Formal System", en Kant-Studien, L (1958-1959). 